\title{
Nuclear PRMT5, cyclin D1 and IL-6 are associated with poor outcome in oropharyngeal squamous cell carcinoma patients and is inversely associated with p16-status
}

\author{
Bhavna Kumar ${ }^{1,2}$, Arti Yadav ${ }^{2}$, Nicole V. Brown ${ }^{3}$, Songzhu Zhao ${ }^{3}$, Michael J. Cipolla ${ }^{1}$, \\ Paul E. Wakely , Alessandra C. Schmitt ${ }^{4,6}$, Robert A. Baiocchi' ${ }^{5}$, Theodoros N. Teknos ${ }^{1,2}$, \\ Matthew Old ${ }^{1,2, *}$, Pawan Kumar ${ }^{1,2, *}$ \\ ${ }^{1}$ Department of Otolaryngology-Head and Neck Surgery, The Ohio State University, Columbus, OH 43210 USA \\ ${ }^{2}$ The Ohio State University Comprehensive Cancer Center, Columbus, OH 43210 USA \\ ${ }^{3}$ Center for Biostatistics, The Ohio State University, Columbus, OH 43210 USA \\ ${ }^{4}$ Department of Pathology, The Ohio State University, Columbus, OH 43210 USA \\ ${ }^{5}$ Department of Internal Medicine, The Ohio State University, Columbus, OH 43210 USA \\ ${ }^{6}$ Department of Pathology and Laboratory Medicine, Emory University School of Medicine, Atlanta, GA 30303 USA \\ *These authors contributed equally to this work
}

Correspondence to: Pawan Kumar, email: Pawan.Kumar@osumc.edu

Keywords: PRMT5, OPSCC, HPV, cyclin D1, IL-6

Received: July 22, $2016 \quad$ Accepted: December 27, $2016 \quad$ Published: January 17, 2017

\section{ABSTRACT}

Protein arginine methyltransferase-5 (PRMT5) plays an important role in cancer progression by repressing the expression of key tumor suppressor genes via the methylation of transcriptional factors and chromatin-associated proteins. However, very little is known about the expression and biological role of PRMT5 in head and neck cancer. In this study, we examined expression profile of PRMT5 at subcellular levels in oropharyngeal squamous cell carcinoma (OPSCC) and assessed its correlation with disease progression and patient outcome. Our results show that nuclear PRMT5 was associated with poor overall survival $(p<0.012)$ and these patients had 1.732 times higher hazard of death (95\% CI: 1.127-2.661) as compared to patients in whom PRMT5 was not present in the nucleus of the tumors. Nuclear PRMT5 expression was inversely correlated with p16-status $(p<0.001)$ and was significantly higher in tumor samples from patients who smoked $>10$ pack-years $(p=0.013)$. In addition, nuclear PRMT5 was directly correlated with cyclin D1 $(p=0.0101)$ and IL-6 expression $(p<0.001)$. In a subgroup survival analysis, nuclear PRMT5-positive/IL-6-positive group had worst survival, whereas nuclear PRMT5-negative/IL-6-negative group had the best survival. Similarly, patients with p16-negative/nuclear PRMT5-positive tumors had worse survival compared to patients with p16-positive/nuclear PRMT5-negative tumors. Our mechanistic results suggest that IL-6 promotes nuclear translocation of PRMT5. Taken together, our results demonstrate for the first time that nuclear PRMT5 expression is associated with poor clinical outcome in OPSCC patients and IL-6 plays a role in the nuclear translocation of PRMT5.

\section{INTRODUCTION}

Head and neck squamous cell carcinoma (HNSCC) is the eighth most frequent cancer worldwide and five-year survival rate $(<50 \%)$ is among the lowest of the major cancers $[1,2]$. Tobacco usage and alcohol consumption have been known to be the strongest risk factors for the development of this disease [3]. However, it is now being recognized that human papillomavirus (HPV) can also play a role in the development of a subset of head and neck cancers [4]. Most of the HNSCC are diagnosed in advanced stages and the outcome of these patients is 
often poor [5]. Five year survival rates for patients with early stage localized head and neck cancers are more than $80 \%$, but drop to $40 \%$ when the disease has spread to the neck nodes, and to below $20 \%$ for patients with distant metastatic disease [5-7]. Patients with head and neck cancers encompass a heterogeneous group and can be further subdivided into two distinct tumor subtypes; human papillomavirus (HPV)-negative and HPV-positive tumors [8]. HPV-positive tumors predominantly arise in the oropharynx and majority of these patients with HPVpositive tumors respond very well to traditional chemoradiotherapy and demonstrate significantly favorable clinical outcomes $[9,10]$. However, there is a small subset of HPV-positive patients that do not respond well to standard therapy and show markedly poor clinical outcome $[11,12]$. In contrast to HPV-positive patients, the majority of HPV-negative patients are usually smokers, have more aggressive disease and many of these patients develop resistance to chemotherapy leading to poor prognosis [10]. Therefore, it is imperative that novel therapeutic targets are identified to increase the long-term survival of these patients as well as decrease the adverse effects associated with standard chemo-radiation regimens [13].

Recent studies have shown that protein arginine methyltransferases (PRMTs) regulate a number of cellular processes, including proliferation, apoptosis, anoikis and epithelial-mesenchymal transition (EMT), which play an important role in tumor growth and metastasis [14-18]. There are nine members in the PRMT family in humans which are subdivided into 3 types based on their distinct methylation characteristics [19]. PRMT2, $3,6,7$ and 8 exhibit tissue specific expression, whereas PRMT1, 4 and 5 are more universally expressed [20]. PRMT5 is the major type II enzyme that mediates posttranslational arginine symmetric dimethylation and it was originally identified as a transcriptional repressor [19, 21]. PRMT5 methylates a host of transcriptional factors (E2F1, p53, FEN1, HOXA9, RAD9 etc.) and chromatinassociated proteins (H2AR3, H3R2, H3R8, H4R3 etc.) to mediate its oncogenic function by repressing the expression of regulatory suppressor genes [18, 22-26]. The transcriptional repressor function of PRMT5 is also critical for the tumor cell release from the primary tumor through the epithelial-mesenchymal transition (EMT) and tumor metastasis. A hallmark of EMT is the loss of E-cadherin expression, which in turn is regulated by the transcription factor Snail. Recently, PRMT5 was shown to repress E-cadherin expression by interacting with Snail through a bridging molecule Ajuba [17]. PRMT5 also regulates cell cycle progression by increasing the expression of positive regulators of G1 phase (Cyclin D1, cyclin D2 and CDK4) and decreasing the expression of negative regulators of G1 (Rb)[27]. Recent studies have shown that PRMT5 is overexpressed in a number of cancer types including ovarian, lung, colon, gastric and bladder cancer and is associated with poor clinical outcome [28-30]. However, there is no reported study that has examined PRMT5 expression, particularly subcellular localization, in a large cohort of tumors from head and neck cancer patient population and its correlation with patient outcome.

In this study, we examined the expression and localization of PRMT5 in oropharyngeal squamous cell carcinoma (OPSCC) tumors and correlated it with patient survival, p16 status (an established surrogate for tumor HPV status in OPSCC), smoking status, cyclin D1 expression, IL-6 expression and other clinical and pathological variables. Our results show a direct correlation between nuclear PRMT5 expression and poor overall survival. Nuclear PRMT5 expression was significantly higher in tumor samples from patients that smoked $>10$ pack-years and inversely correlated with p16 status. In addition, nuclear PRMT5 expression directly correlated with IL-6 and cyclin D1 expression. In our mechanistic experiments, IL-6 treatment markedly enhanced nuclear translocation of PRMT5 in head and neck cancer cells.

\section{RESULTS}

To evaluate the expression pattern and clinical importance of PRMT5, cyclin D1 and IL-6 in OPSCC, we assessed the expression of these biomarkers in TMA's constructed using 211 surgically treated samples. Patient characteristics are listed in Table 1. Representative images of staining for PRMT5 and cyclin D1 are included in Figures 1 and 4, respectively. p16 was used as surrogate marker for HPV status. 158 tumors $(75.2 \%)$ were p16 positive and 52 tumors $(24.8 \%)$ were negative. The median follow-up time was 5.5 years (range $0.1-11.5$ ). The 5-year survival rates for the whole group were $60.6 \%$; $69.6 \%$ for the p16-positive group and $32.6 \%$ for the $\mathrm{p} 16$ negative group.

\section{Nuclear PRMT5 expression is directly associated with poor overall survival and inversely associated with p16 status}

PRMT5 expression was evaluable in 209 tumors and it was predominately cytoplasmic in the OPSCC tumor samples $(72.2 \% ; 151 / 209)$. However, we also observed nuclear PRMT5 expression in 58/209 (27.8\%) tumors. Tumors that expressed PRMT5 in the nucleus were categorized as nuclear positive and tumors with cytoplasmic expression were classified as nuclear negative. Higher nuclear PRMT5 expression was associated with poor overall survival $(p<0.012)$. Patients whose tumors were nuclear PRMT5 positive had a 1.732 hazard ratio of death (95\% CI: 1.127-2.661) as compared to those in which PRMT5 was not present in the nucleus (Figure 1A-1B). The poorer prognosis of patients with tumors expressing nuclear PRMT5 was maintained after adjustments for age, 
Table 1: Patient Characteristics and Demographics

\begin{tabular}{|c|c|c|}
\hline Patient Characteristics & $n$ & $\%$ \\
\hline Age (years), mean (SD) & 57.7 & 9.7 \\
\hline \multicolumn{3}{|l|}{ Marital Status } \\
\hline Single/Divorced/Widowed & 87 & 45.6 \\
\hline Married & 104 & 54.5 \\
\hline \multicolumn{3}{|l|}{ Race } \\
\hline African American/Black & 9 & 4.3 \\
\hline White & 202 & 95.7 \\
\hline \multicolumn{3}{|l|}{ Sex } \\
\hline Female & 45 & 21.3 \\
\hline Male & 166 & 78.7 \\
\hline \multicolumn{3}{|l|}{ Smoking Status } \\
\hline$\leq 10$ pack years & 51 & 25.1 \\
\hline$>10$ pack years & 152 & 74.9 \\
\hline \multicolumn{3}{|l|}{ Extracapsular Spread } \\
\hline No & 118 & 57.8 \\
\hline Yes & 86 & 42.2 \\
\hline \multicolumn{3}{|l|}{ p16 Status } \\
\hline Negative & 52 & 24.8 \\
\hline Positive & 158 & 75.2 \\
\hline \multicolumn{3}{|l|}{ Mucosal Margins } \\
\hline Free of Carcinoma & 174 & 83.7 \\
\hline Positive Margins & 34 & 16.4 \\
\hline \multicolumn{3}{|l|}{ Primary site } \\
\hline BOT & 52 & 24.8 \\
\hline Tonsil & 141 & 67.1 \\
\hline Other & 17 & 8.1 \\
\hline \multicolumn{3}{|l|}{ Node Stage } \\
\hline N0 & 28 & 13.3 \\
\hline N1 & 48 & 22.8 \\
\hline $\mathrm{N} 2$ & 126 & 59.7 \\
\hline $\mathrm{N} 3$ & 9 & 4.3 \\
\hline \multicolumn{3}{|l|}{ Perineural Invasion } \\
\hline No & 157 & 74.8 \\
\hline Yes & 53 & 25.2 \\
\hline \multicolumn{3}{|l|}{ AJCC Stage } \\
\hline I & 5 & 2.4 \\
\hline II & 11 & 5.2 \\
\hline IIII & 51 & 24.2 \\
\hline $\mathrm{IV}$ & 144 & 68.3 \\
\hline \multicolumn{3}{|l|}{\begin{tabular}{|l} 
Tumor Stage \\
\end{tabular}} \\
\hline $\mathrm{T} 1$ & 45 & 21.3 \\
\hline $\mathrm{T} 2$ & 86 & 40.8 \\
\hline $\mathrm{T} 3$ & 41 & 19.4 \\
\hline $\mathrm{T} 4$ & 39 & 18.5 \\
\hline
\end{tabular}


T stage, $\mathrm{N}$ stage, AJCC stage, gender and smoking status ( $p=0.0055$; HR 1.912 95\% CI: 1.210-3.022). We and others have previously shown that OPSCC patients with HPV-negative tumors have inferior outcomes as compared to patients with HPV-positive patients $[9,10]$. In this present study, we also observed that patients with p16-negative tumors have significantly higher hazard of death (HR: 2.76; 95\% CI 1.826-4.184) as compared to patients with p16positive tumors (Figure 2A). This poor prognosis of patients with p16-negative tumors was maintained after adjustments for age, T stage, N stage, AJCC stage, gender and smoking status ( $p=0.0043$; HR 2.073 95\% CI:1.257-3.418). Nuclear PRMT5 expression was inversely associated with p16 expression. A greater proportion of p16-negative tumors expressed higher nuclear PRMT5 as compared to p16-positive tumors $(58.65 \%$ versus 16.35 , Figure $2 \mathrm{~B}$, $p<0.001)$. In the subgroup survival analysis, $\mathrm{p} 16$-negative/ PRMT5 nuclear-positive (p16-/Nuclear PRMT5+) group had the worst survival compared to the other groups (Figure 2C, $p<0.001$ ).

Patients who smoked $>10$ pack-year showed poor overall survival and had significantly higher expression of nuclear PRMT5. We and others have previously shown that smokers have poor clinical outcome as compared to non-smokers $[10,31]$. In this study, our results further corroborate those finding and show that patients who smoked $>10$ pack-year had significantly poor overall survival as compared to patients who smoked $\leq 10$ pack-year ( $p=0.046$, Figure 3A). The hazard of death for patients who smoked $>10$ pack-years was 1.691 (95\% CI: 1.009-2.832). In addition, smokers ( $>10$ packyears) had significantly higher nuclear PRMT5 expression
(Figure $3 \mathrm{~B}, p=0.013$ ). A larger proportion of patients who smoked $>10$ pack-years were positive for nuclear PRMT5 expression as compared to patients that smoked $\leq 10$ pack-years $(24.38 \%$ versus $3.98 \%)$. In the subgroup survival analysis, PRMT5 nuclear-negative/ $\leq 10$ pack years group had the best survival compared to the other groups (Figure 3C, $p=0.0107$ ).

\section{Cyclin D1 expression is associated with poor overall survival and is directly correlated with nuclear PRMT5 expression}

Recent studies have shown that PRMT5 regulates tumor growth by modulating cyclin D1 expression [27]. In addition, amplification of cyclin D1 gene and overexpression of cyclin D1 protein has been reported in HNSCC [32]. Cyclin D1 expression was evaluable in 197 OPSCC tumors. Patients with tumor that overexpressed cyclin D1 showed poor overall survival as compared to patients with tumor that were negative for cyclin D1 expression (Figure 4A-4B). The hazard of death for patients with positive cyclin D1 expression was 2.025 (95\% CI: 1.326-3.092). This poorer prognosis of patients with tumors expressing higher cyclin D1 was maintained after adjustments for age, T stage, $\mathrm{N}$ stage, AJCC stage, gender and smoking status $(p=0.0009$; HR 2.209 95\% CI: 1.385-3.523). Patients whose tumors were nuclear PRMT5 positive had higher expression of cyclin D1 ( $p=0.01$, Figure 5A). Similarly, patients whose tumors were p16-negative had higher expression of cyclin D1 $(p<.001$, Figure 5B). In the subgroup survival analysis, PRMT5 nuclear-positive and cyclin D1-positive (nuclear

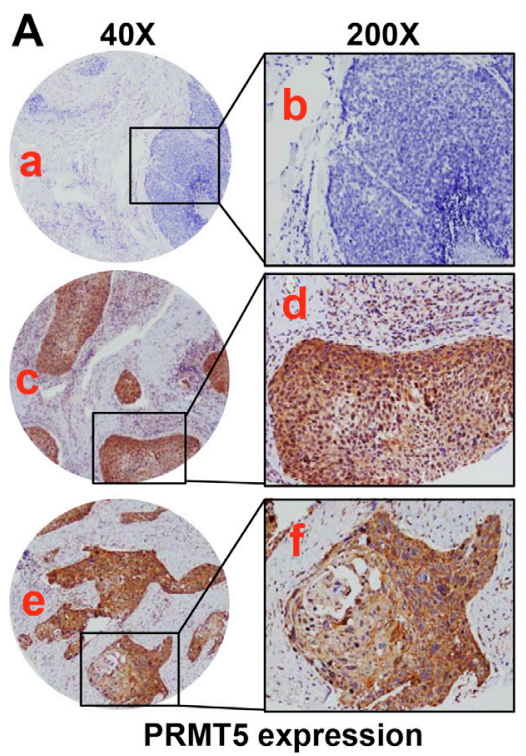

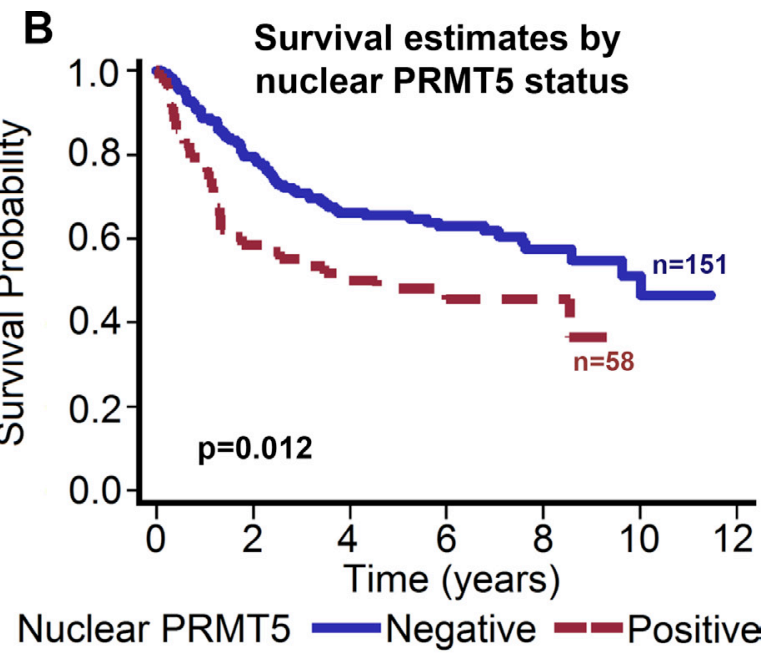

Patients with nuclear PRMT5 have increased hazard of death $\mathrm{HR}: 1.73 ; 95 \% \mathrm{Cl}(1.13-2.66)$

Figure 1: Nuclear PRMT5 expression is associated with poor overall survival in OPSCC. Tissue microarrays (TMAs) containing primary tumor samples from OPSCC patients were stained for PRMT5 expression. (A) Representative pictures of tumor cores negative for PRMT5 expression ( $a$ and b), positive for nuclear PRMT5 expression ( $c$ and $d$ ), and negative for nuclear PRMT5 expression (positive for cytosolic PRMT5 expression, e and f). (B) Overall survival estimates of patients according to nuclear PRMT5 expression. 
PRMT5+/cyclin D1+) group had the worst survival compared to the other groups (Figure 5C). In addition, cyclin D1 expression was directly associated with tumor recurrence $(p=0.04)$.

\section{IL-6 overexpression is associated with poor overall survival and is directly correlated with nuclear PRMT5 expression}

IL-6 expression was evaluable in 198 tumors and was found to be overexpressed in 112 (56.6\%) tumors. Overexpression of IL-6 was significantly associated with worse overall survival of patients with OPSCC $(p<0.001)$ [33]. The hazard of death for patients with positive IL-6 expression was 3.8 (95\% CI: 2.287-6.324, Figure 6A). This poorer prognosis of patients with tumors expressing higher IL-6 was maintained after adjustments for age, T stage, N stage, AJCC stage, gender and smoking status ( $p<0.0001$; HR 4.089 95\% CI: 2.390-6.995). Interestingly, nuclear PRMT5 expression was directly associated with high IL-6 expression in the primary tumor samples $(p<0.001$; Figure 6B). In the subgroup survival analysis, IL-6+/nuclear PRMT5+ group had the worst survival as compared to IL-6-/nuclearPRMT5+ and
IL-6-/nuclear PRMT5- groups ( $p<0.001$, Figure 6C). In addition, IL-6 overexpression was also directly associated with tumor recurrence $(p<0.001)$, perineural invasion $(p=0.007)$, extracapsular spread $(p=0.014)$ and inversely associated with p16 status $(p=0.011)$.

\section{IL-6 promotes nuclear translocation of PRMT5}

Our results from this study show that nuclear localization of PRMT5 was directly associated with poor clinical outcome in OPSCC patients. In the same patient cohort, IL-6 overexpression was also associated with poor survival. Interestingly, high expression of IL-6 was directly correlated with nuclear PRMT5 localization, thereby suggesting that IL-6 might be promoting PRMT5 nuclear translocation. To examine the effect of IL-6 on PRMT5 nuclear localization, we performed nuclear/ cytosolic fractionation and immunofluorescence staining experiments using head and neck cancer cell lines. Our results show that IL-6 treatment of CAL27 cells markedly enhanced nuclear translocation of PRMT5 (Figure 7A-7B). Similar IL-6-mediated nuclear translocation of PRMT5 was observed in another head and neck cell line (UM-SCC74B; Figure 7C).
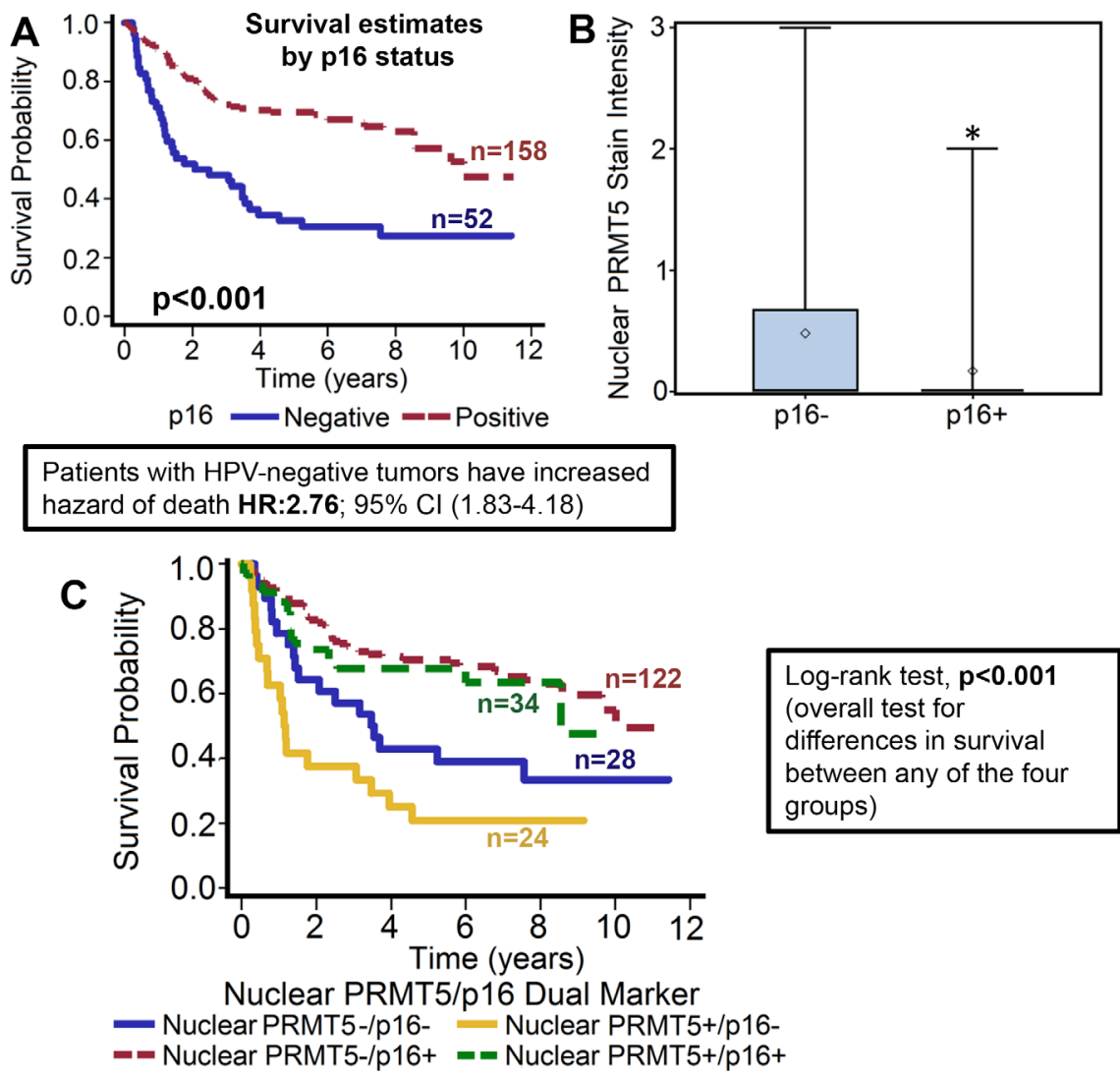

Figure 2: Nuclear PRMT5 expression is significantly lower in p16-positive tumors as compared to p16-negative tumors. (A) Overall survival estimates of patients according to p16 status. (B) Nuclear PRMT5 expression in p16-negative versus p16positive tumors. *represent a significant difference in nuclear PRMT5 expression in p16-positive (p16+) tumor samples as compared to p16-negative (p16-) tumor samples. (C) Survival estimates of patients according to nuclear PRMT5 expression and p16 status. 

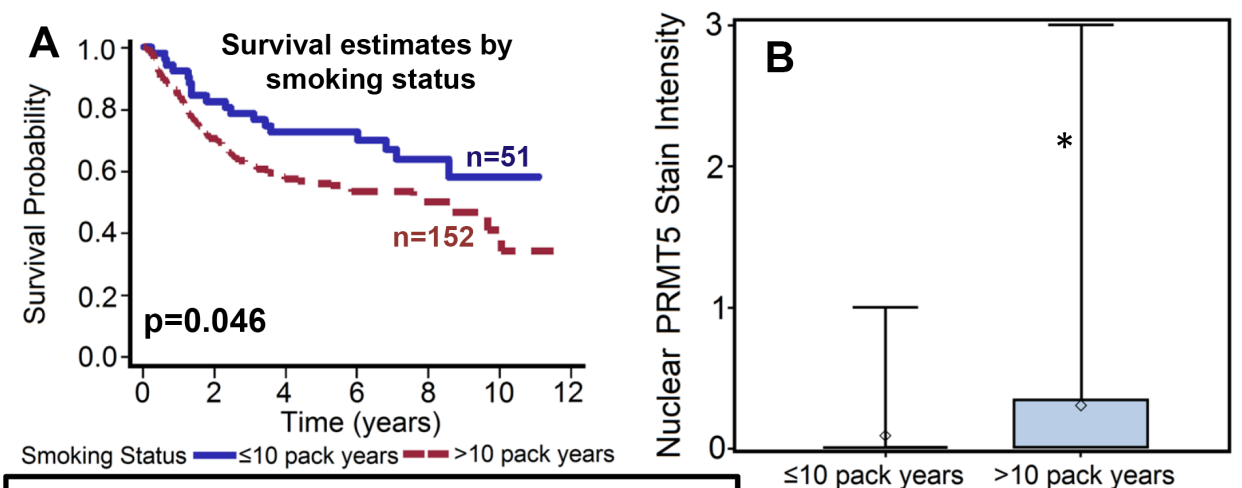

Patients who smoked $>10$ pack-years have increased hazard of death $\mathrm{HR}: 1.691 ; 95 \% \mathrm{Cl}(1.01-2.83)$

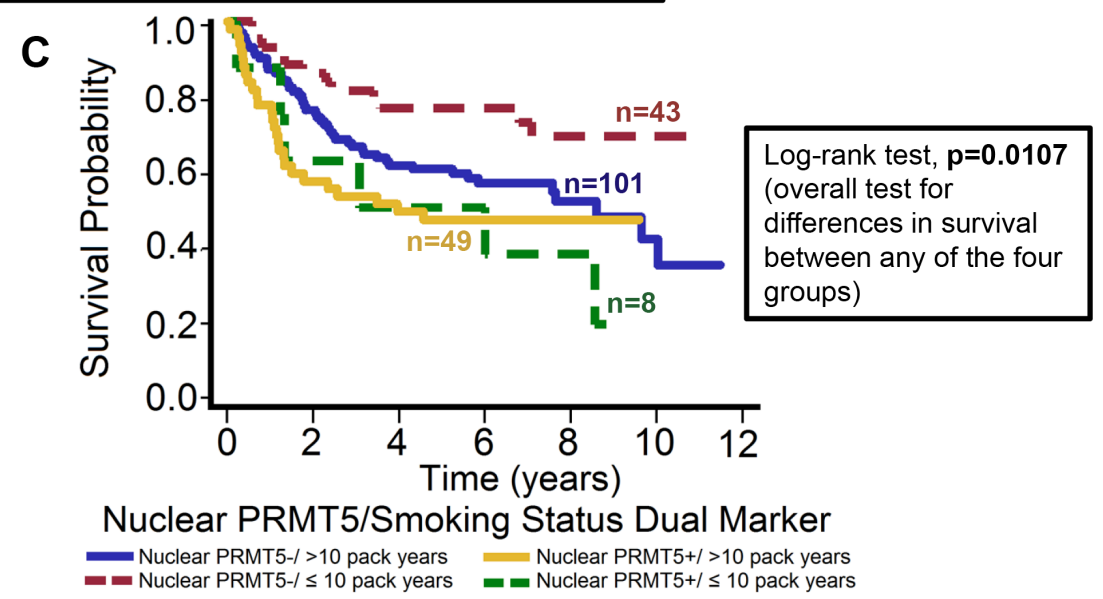

Figure 3: Patients who smoked $>10$ pack-years had significantly poor overall survival and markedly higher nuclear PRMT5 expression. (A) Overall survival estimates of patients according to smoking status. (B) Nuclear PRMT5 expression in tumor samples from patients that smoked $\leq 10$ pack-years or $>10$ pack-years. *represent a significant difference in nuclear PRMT5 expression in patients that smoked $<10$ pack-years as compared patients that smoked $>10$ pack-years. $(\mathbf{C})$ Overall survival estimates according to nuclear PRMT5 expression and smoking status.
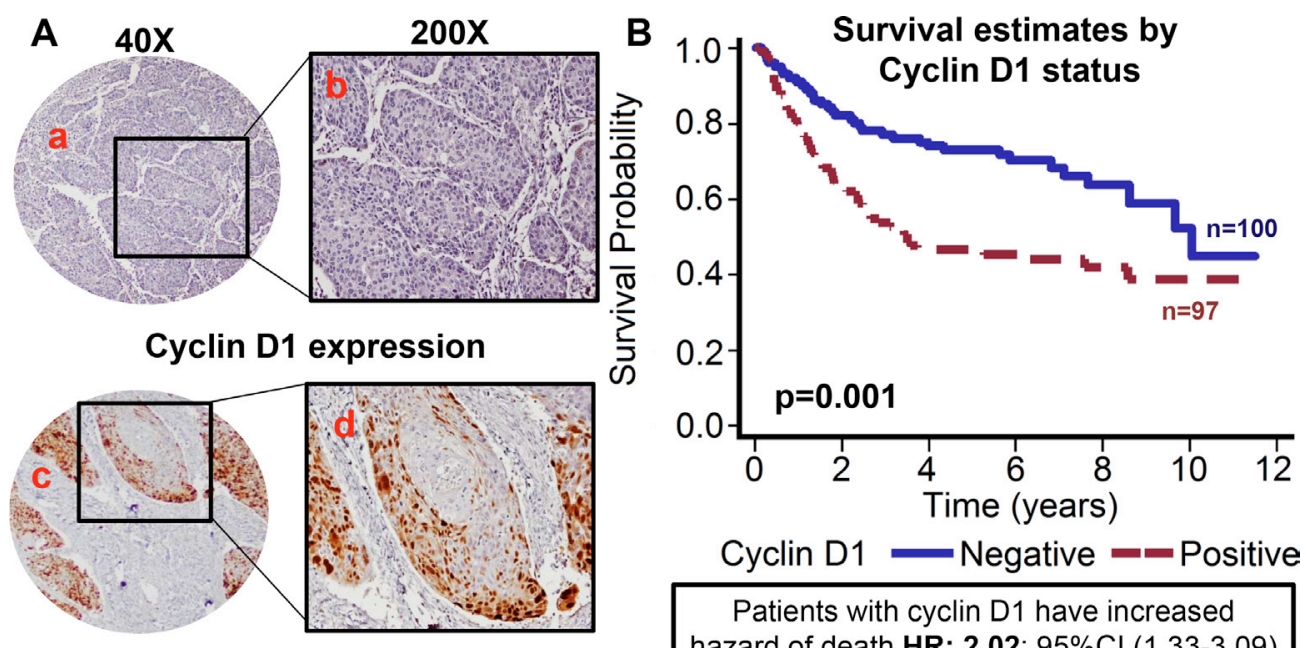

$$
\begin{array}{|c|}
\text { Cyclin D1 Negative } \\
\hline \text { Patients with cyclin D1 have increased } \\
\text { hazard of death HR: } 2.02 ; 95 \% \mathrm{Cl}(1.33-3.09)
\end{array}
$$

Figure 4: Cyclin D1 expression is associated with poor overall survival. TMAs were stained for cyclin D1 expression. (A) Representative pictures of tumor cores negative for cyclin D1 expression (a and b) and positive for cyclin D1 expression (c and d). (B) Overall survival estimates of patients according to cyclin D1 expression. 


\section{DISCUSSION}

The role of PRMT5 in cancer, particularly in regulating the expression of key tumor suppressor genes, has been extensively studied $[14,15,18]$. However, there are conflicting reports about the significance of nuclear versus cytoplasmic localization of PRMT5. In this study, we examined the PRMT5 subcellular expression pattern in surgically treated oropharyngeal squamous cell carcinoma (OPSCC) samples and correlated that with survival, p16 status, and other clinical and pathological variables. Our results show that nuclear PRMT5 expression was highly predictive of poor overall survival in OPSCC patients and it directly correlated with cyclin D1 and IL-6 expression. Nuclear PRMT5 expression has been found to be directly associated with invasive colorectal carcinoma and highly proliferative breast cancer [34, 35]. In contrast, cytoplasmic PRMT5 expression was recently shown to be directly correlated with poor prognosis in lung adenocarcinoma [36, 37]. It is well established that PRMT5 mediates its biological effects by methylating a number of different proteins including p53, myelin basic protein and SM proteins [38-40]. However, it is the histone dimethylation, particularly symmetric dimethylation of histone 4(R3) and histone 3(R8), that is associated with transcriptional repression [16, 18]. Histones are exclusively localized and perform their biological functions in the nucleus of eukaryotic cells. Therefore, PRMT5 translocation to nucleus is essential for histone methylation and subsequent repression of tumor suppressor genes. We had recently shown that IL-6 overexpression promotes tumor metastasis in head and neck cancer by inducing epithelial-mesenchymal transition (EMT) [41]. In this study, we observed that nuclear PRMT5 expression is directly correlated with high IL-6 expression, thereby suggesting that IL-6 might be promoting PRMT5 translocation to nucleus. Indeed, our mechanistic experiments showed that IL-6 treatment of head and neck cancer cells promote PRMT5 translocation to nucleus. PRMT5 subcellular localization is also regulated by binding partners. Recently, IL-6 target protein Snail was shown to form a complex with PRMT5-MEP50. This complex was translocated to the nucleus by interaction with LIM protein Ajuba [17]. Snail recruits this complex to the E-cadherin proximal promoter site, resulting increased H4R3 methylation. These results

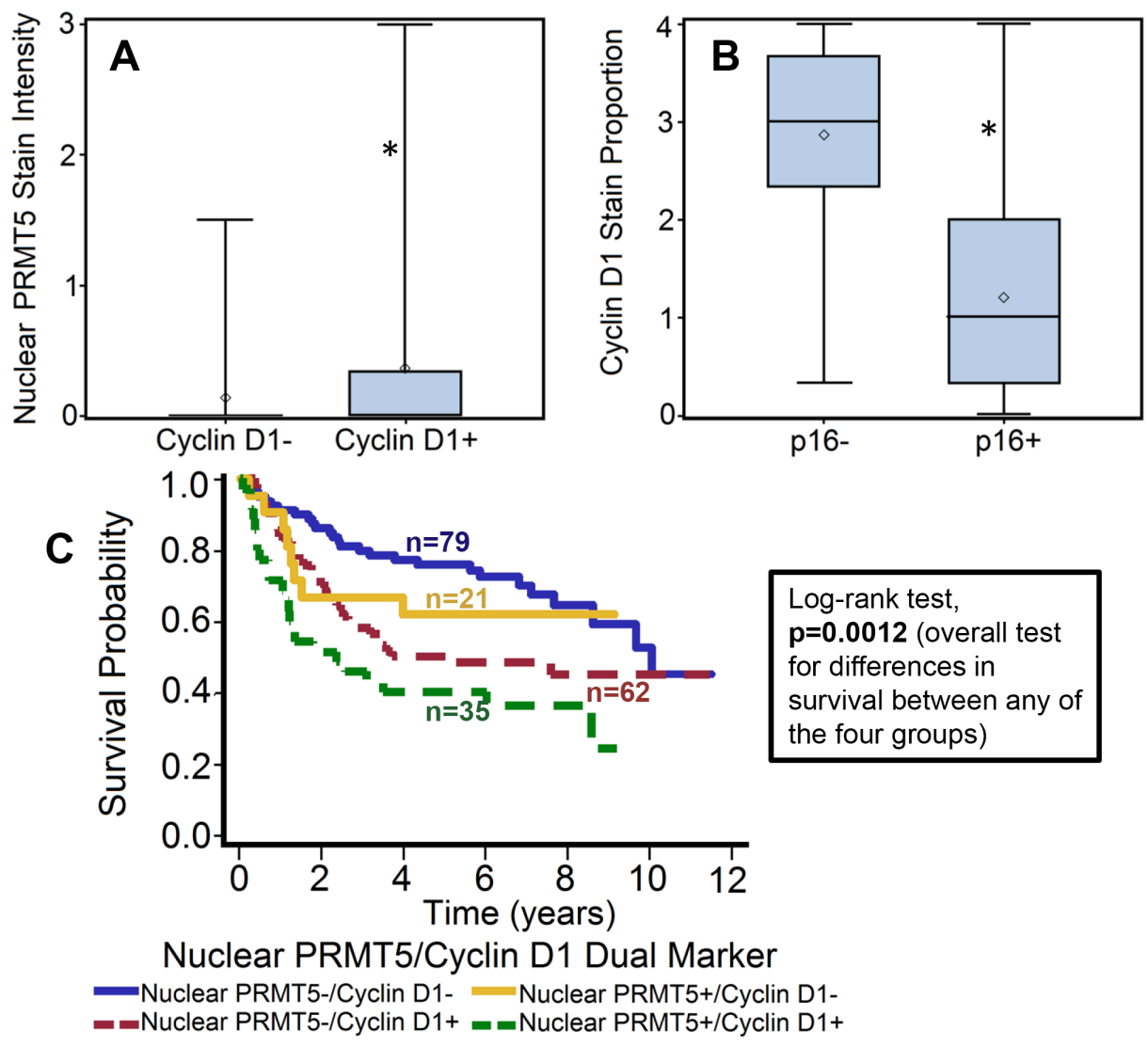

Figure 5: Cyclin D1 expression is directly correlated with nuclear PRMT5 expression and inversely with p16 status. (A) Nuclear PRMT5 expression in tumor samples with cyclin D1-negative (cyclin D1-) or cyclin D1-positive (cyclin D1+) expression. *represent a significant difference in nuclear PRMT5 expression in tumor samples with cyclin D1-positive or cyclin D1-negative expression. (B) Cyclin D1 expression in p16-negative versus p16-positive tumors. *represent a significant difference in cyclin D1 expression in tumor samples with p-positive as compared to p-negative tumors. (C) Overall survival estimates according to nuclear PRMT5 and cyclin expression. 

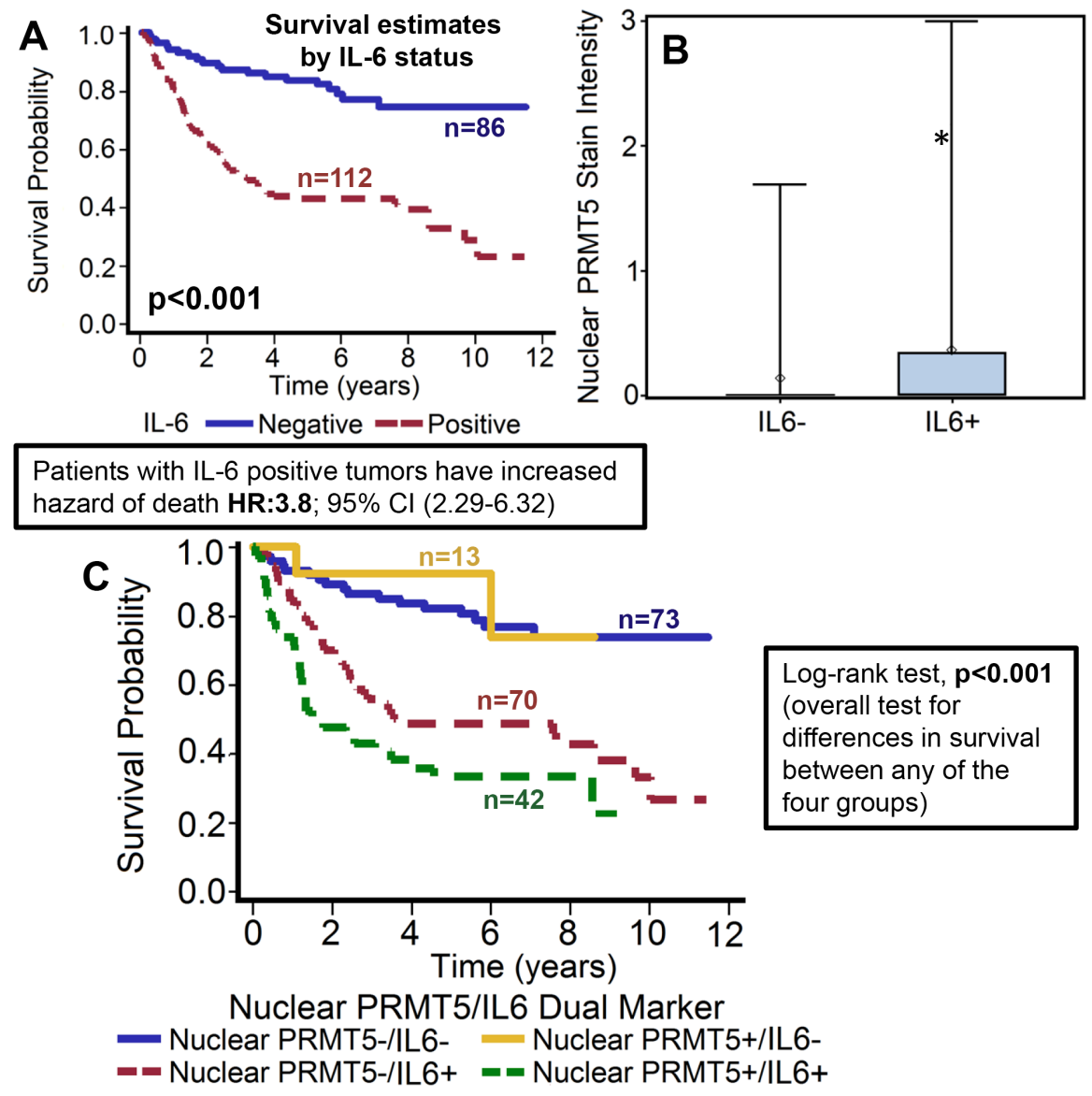

Figure 6: High IL-6 expression is associated with poor overall survival and directly correlates with nuclear PRMT5 expression. (A) Overall survival estimates of patients according to IL-6 expression. (B) Nuclear PRMT5 expression in IL-6-negative (IL-6-) or IL-6-positive (IL-6+) tumor samples. (C) Overall survival estimates according to nuclear PRMT5 expression and IL-6 expression.
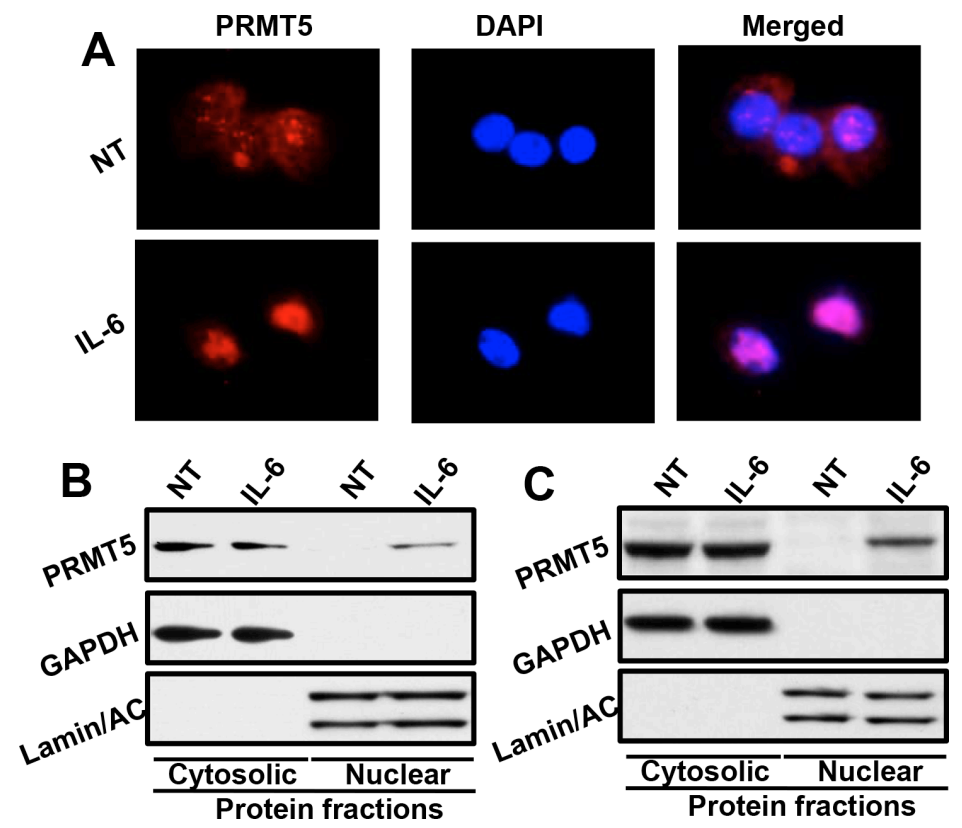

Figure 7: IL-6 promotes PRMT5 nuclear translocation. (A) CAL27 cells were cultured in Labtech chambers and treated with IL-6 for 30 minutes. Cells were then stained for PRMT5 (red) and nucleus (DAPI, blue) and photographed at 600X. (B-C) CAL27 (B) or UM-SCC-74B (C) cells were treated with IL-6 for 30 minutes. Nuclear and cytosolic fractions were isolated from these cells and Western blotted for GAPDH, Lamin A/C and PRMT5. 
suggest that transcriptional repression of E-cadherin and EMT by IL-6/snail is dependent on nuclear translocation of PRMT5 and its methyltransferase activity [41]. A number of studies have shown that IL-6 promotes head and neck cancer cell proliferation, migration, survival, invasion, epithelial to mesenchymal transition (EMT), stem cell expansion, and chemoresistance via the activation of JAK/STAT3 signaling pathway [41-43]. Recently, JAK2V617F (a constitutively active JAK2 mutant) was shown to phosphorylates PRMT5 in myeloproliferative neoplasms [44]. It is possible that PRMT5 nuclear translocation is promoted by IL-6/JAK2 mediated PRMT5 phosphorylation.

Over the past decade, it has become apparent that the incidence of "classic" tobacco/alcohol-induced HNSCC has declined, but at the same time, HNSCC caused by HPV has risen sharply [45]. HPV16 is the most prevalent subtype and it accounts for $\approx 90 \%$ of HPV-related HNSCC [46, 47]. Intriguingly, patients with HPV-related HNSCC tend to have far better prognosis than HPV-negative counterparts $[9,10]$. A number of potential models have been proposed to explain this clinical outcome disparity between HPV-positive versus HPV-negative patients. However, we still know very little about the precise molecular mechanism(s) that could explain this clinical outcome disparity. In this study, we show an inverse correlation between p16-positivity and nuclear PRMT5 expression. These results suggest that differential nuclear PRMT5 expression might be one of the contributing factors for this prognosis disparity in HPV-positive versus HPV-negative patients. It is possible that high expression of nuclear PRMT5 in HPV-negative tumors is due to the high expression of IL-6 in these tumors as compared to HPV-positive tumors [48].

Cyclin D1 is a key regulator of cell cycle and is often overexpressed in numerous cancer types including breast, colon, lung and head and neck [32, 49]. Our results suggest that high cyclin D1 expression is correlated with poor patient outcome and directly associated with nuclear PRMT5 expression. This could be due to PRMT5-mediated enhanced expression of positive regulators of $\mathrm{G} 1$ phase including cyclin D1 [27]. There seems to be a positive feed-back loop between PRMT5 activity and cyclin D1. In addition to PRMT5 increasing cyclin D1 expression, cyclin D1 in turn enhances PRMT5 methyltransferase activity by phosphorylating MEP50 that leads to the formation of MEP50-PRMT5 complex [49]. Taken together, our results suggest that nuclear PRMT5 is a marker for poor clinical outcome in OPSCC. Our mechanistic studies suggest that IL-6 promotes PRMT5 translocation to nucleus. Inhibitors designed to block PRMT5 nuclear translocation and its transcriptional regulatory function may lead to the development of novel therapeutic strategies for the treatment of head and neck cancer.

\section{MATERIALS AND METHODS}

\section{Study population}

Oropharyngeal squamous cell carcinoma (OPSCC) tissue specimens were obtained from surgical resections of patients at The Ohio State University James Cancer Hospital and Solove Research Institute between 2002 to 2009. All patients underwent surgical resection as a first line of therapy with a curative intent. This was followed by no additional treatment or adjuvant chemotherapy and/ or radiotherapy as needed. The Ohio State University Institutional Review Board approved a retrospective analysis study of these specimens and a waiver of HIPAA authorization was obtained. Patient characteristics, including age, race, gender, marital status, smoking status, pathological variables including tumor size, nodal status, AJCC stage, extracapsular spread, perineural invasion and clinical variables including survival and recurrence outcomes were recorded.

\section{Tissue microarray (TMA)}

Paraffin-embedded archival tissue blocks and their matching H\&E-stained slides were retrieved from the Department of Pathology. A pathologist marked the areas with cancer and adjacent normal on the H\&E slides. Representative regions (three cores of tumor tissue and one core of adjacent normal tissue) were sampled using a 0.6-mm punch on a master TMA blocks. The TMA's were constructed by the Histology Core in the Department of Pathology. Unstained sections were cut and used for immunohistochemical staining.

\section{Immunohistochemistry and scoring}

TMA slides were stained to assess the tumor expression of PRMT5, p16, cyclin D1 and IL-6, using immunohistochemistry as previously described [10]. Briefly, slides were deparaffinized in xylene, and rehydrated in decreasing concentrations of ethyl alcohol. Antigen retrieval was performed in a decloaking chamber (Biocare Medical, LLC, Concord, CA, USA) using antigen unmasking buffer (Dako) for 20 minutes at $120^{\circ} \mathrm{C}$. After a 20 minutes cool down period at room temperature, sections were incubated with dual endogenous enzyme block (Dako) for 10 minutes at room temperature. Nonspecific binding sites were blocked by incubating with PBS /serum from species in which the secondary antibody was raised. Sections were then incubated with primary antibody (IL-6 for overnight; R\&D Systems, PRMT5 for 1 hour; Cell Signaling and Cyclin D1 for 1 hour; Thermo Scientific-Lab Vision) at room temperature. After washes, slides were incubated with biotinylated donkey anti-goat (Jackson Immunoresearch; for IL-6) or, biotinylated anti-rabbit (Vectastain Elite Kit; for PRMT5 and Cyclin 
D1) for 30 minutes at room temperature. Slides were rinsed in wash buffer and incubated with avidin-biotin complex (Vector Laboratories, Burlingame, CA, USA) for 30 minutes. They were then rinsed in wash buffer and incubated with 3,3'-diaminobenzidine (Sigma). The slides were rinsed in water, counterstained with Mayer's hematoxylin, mounted and coverslipped. p16 expression was determined using the CINtec p16 histology kit (MTM/Roche, Heidelberg, Germany). Stained slides were interpreted by a pathologist who was blinded to treatment outcome at the time of review. For p16, cyclin D1 and IL-6, each core was scored for the stain proportion $(0-100 \%)$ and intensity (1: none, 2 : low, 3 : moderate, 4: high). A quick score was generated by multiplying the stain proportion scores with stain intensity scores to obtain values between 0-400. For PRMT5 expression, cores were scored for stain intensity and location of PRMT5 (nuclear, cytoplasmic or none). A tumor was considered to be p16 positive when $\geq 50 \%$ of the tumor cells displayed strong and diffuse staining pattern.

\section{Cell lines and reagents}

CAL27 cells were obtained from ATCC (Manassas, VA). UM-SCC-74B cell line was obtained from the laboratory of Dr. Thomas E. Carey at the University of Michigan.[50] The identity of both the tumor cell lines was confirmed by STR genotyping (AmpFLSTR Identifiler Kit, Applied Biosystems, Carlsband, CA). The tumor cell lines were cultured in DMEM supplemented with $10 \%$ fetal bovine serum containing $1 \%$ penicillin/ streptomycin (Invitrogen, Carlsbad, CA) and 1\% Nonessential amino acids. Recombinant IL-6 was obtained from PeproTech (Rocky Hill, NJ). Primary antibodies against PRMT5 and Lamin/AC were purchased from Cell Signaling (Danvers, MA) and GAPDH was purchased from Millipore (Billerica, MA).

\section{Cytoplasmic and nuclear protein extraction}

Cytoplasmic and nuclear fractions from tumor cells were separated using the NE-PER Nuclear \& Cytoplasmic extraction kit (Pierce, Rockford, IL). After IL-6 treatment, cells were harvested and washed with PBS. Cell pellet was treated with cell membrane lysis reagents from the extraction kit to release the cytoplasmic contents. The cytoplasmic proteins were collected by centrifugation leaving the intact nuclei in the pellet. The nuclear pellet was washed with PBS to reduce carryover of the cytoplasmic proteins to the nuclear protein fraction. Nuclear lysis buffer was then added to the pellet to lyse the nuclei and release the nuclear proteins. Reducing agent and loading buffer were then added to the cytoplasmic and nuclear extracts and analyzed by Western blotting.

\section{Western blot analysis}

Whole cell lysates or cytosolic/nuclear protein fractions were separated by 4-12\% NuPAGE Bis-Tris gels (Invitrogen, Carlsbad, CA) and transferred onto PVDF membranes using NuPAGE transfer buffer (Invitrogen). To block nonspecific binding, membranes were incubated with $5 \%$ Milk in Tris buffered saline containing $0.1 \%$ Tween-20 (TBST) or 3\% BSA in TBST for 1 hour at room temperature. The blots were then incubated with the respective primary antibody in TBST $+5 \%$ Milk or $3 \%$ BSA according to manufacturer's instructions at $4^{\circ} \mathrm{C}$ overnight. After washing with TBST, the blots were incubated with horseradish peroxidase-conjugated sheep anti-mouse $\operatorname{IgG}(1: 3,000)$ or with donkey anti-rabbit $\operatorname{IgG}$ $(1: 4,000)$ for 1 hour at room temperature. An ECL-plus Western blotting substrate (Thermo Scientific-Pierce, Rockford, IL) was used to detect specific protein bands.

\section{Immunofluorescent staining}

Tumor cells were cultured in Labtech chambers in serum free medium for 2 hours and then treated with recombinant IL-6 $(50 \mathrm{ng} / \mathrm{ml})$ for 30 minutes. At the end of incubation, cells were fixed with $4 \%$ paraformaldehyde for 15 minutes at room temperature and permeabilized by treating with $100 \%$ methanol for 10 minutes at $-20^{\circ} \mathrm{C}$. Next, slides were washed with PBS, blocked with normal goat IgG for 1 hour and incubated overnight at $4{ }^{\circ} \mathrm{C}$ with mouse anti-PRMT5 antibody. After washing with PBS, chamber slides were incubated with secondary antibodies (goat anti-mouse-IgG-Alexa Fluor 488). Chamber slides were then mounted with ProLong Gold antifade reagent with DAPI (Invitrogen). The fluorescent images were captured using Nikon Eclipse 80i microscope with DS-Ri1 camera at $600 \mathrm{X}$ magnification and overlaid using NISElements-Basic Research software (Nikon, Melville, NY).

\section{Statistical analyses}

Overall survival was defined as time from the date of surgery to date of death, with patients alive at the date of last observation censored. Cox proportional hazards models were used to assess univariate associations of biomarkers or patient characteristics as predictors for death. Unadjusted hazard ratios (HR) and $95 \%$ confidence intervals (CI) are reported. Multivariable models were built to estimate adjusted HRs which let us asses marker effects (nuclear PRMT5, p16, Cyclin D1, and IL-6) beyond the effects of T stage, N stage, AJCC stage, smoking status and gender. To assess dual marker interactions, comparisons of survival curves were evaluated using the log-rank test with adjustments for multiple comparisons made by Bonferonni corrections. Mann-Whitney tests were used to assess associations between biomarkers (p16, Cyclin D1, and IL-6) 
or demographics (smoking status) and nuclear PRMT5 or Cyclin D1 expression. All analyses were conducted in SAS, version 9.3 (SAS Institute, Cary, North Carolina).

\section{CONFLICTS OF INTEREST} interests.

The authors declare that they have no competing

\section{GRANT SUPPORT}

NIH/NCI-CA178649 (P. Kumar) and funds from The Ohio State University Comprehensive Cancer Center.

\section{REFERENCES}

1. Siegel R, Ma J, Zou Z, Jemal A. Cancer statistics, 2014. CA Cancer J Clin. 2014; 64:9-29.

2. Ferlay J, Shin H-R, Bray F, Forman D, Mathers C, Parkin DM. Estimates of worldwide burden of cancer in 2008: GLOBOCAN 2008. International Journal of Cancer. 2010; 127:2893-2917.

3. Sturgis EM, Wei Q, Spitz MR. Descriptive epidemiology and risk factors for head and neck cancer. Semin Oncol. 2004; 31:726-733.

4. Marur S, D'Souza G, Westra WH, Forastiere AA. HPVassociated head and neck cancer: a virus-related cancer epidemic. Lancet Oncol. 2010; 11:781-789.

5. Kalavrezos N, Bhandari R. Current trends and future perspectives in the surgical management of oral cancer. Oral Oncol. 2010; 46:429-432.

6. Ferlito A, Shaha AR, Silver CE, Rinaldo A, Mondin V. Incidence and Sites of Distant Metastases from Head and Neck Cancer. ORL. 2001; 63:202-207.

7. Hauswald H, Simon C, Hecht S, Debus J, Lindel K. Longterm outcome and patterns of failure in patients with advanced head and neck cancer. Radiation Oncology. $2011 ; 6: 70$.

8. Gillison ML, D'Souza G, Westra W, Sugar E, Xiao W, Begum S, Viscidi R. Distinct risk factor profiles for human papillomavirus type 16-positive and human papillomavirus type 16-negative head and neck cancers. J Natl Cancer Inst. 2008; 100:407-420.

9. Fakhry C, Westra WH, Li S, Cmelak A, Ridge JA, Pinto H, Forastiere A, Gillison ML. Improved survival of patients with human papillomavirus-positive head and neck squamous cell carcinoma in a prospective clinical trial. J Natl Cancer Inst. 2008; 100:261-269.

10. Kumar B, Cordell KG, Lee JS, Worden FP, Prince ME, Tran HH, Wolf GT, Urba SG, Chepeha DB, Teknos TN, Eisbruch A, Tsien CI, Taylor JM, et al. EGFR, p16, HPV Titer, Bcl-xL and p53, sex, and smoking as indicators of response to therapy and survival in oropharyngeal cancer. J Clin Oncol. 2008; 26:3128-3137.
11. Kaka AS, Kumar B, Kumar P, Wakely PE, Jr., Kirsch CM, Old MO, Ozer E, Agrawal A, Carrau RE, Schuller DE, Siddiqui F, Teknos TN. Highly aggressive human papillomavirus-related oropharyngeal cancer: clinical, radiologic, and pathologic characteristics. Oral Surg Oral Med Oral Pathol Oral Radiol. 2013; 116:327-335.

12. Quon H, Forastiere AA. Controversies in treatment deintensification of human papillomavirus-associated oropharyngeal carcinomas: should we, how should we, and for whom? J Clin Oncol. 2013; 31:520-522.

13. Allen AM, Elshaikh $\mathrm{M}$, Worden FP, Bradford CR, Teknos TN, Chepeha DB, Tsien C, Dawson LA, Urba S, Wolf GT, Normolle D, Eisbruch A. Acceleration of hyperfractionated chemoradiation regimen for advanced head and neck cancer. Head Neck. 2007; 29:137-142.

14. Stopa N, Krebs J, Shechter D. The PRMT5 arginine methyltransferase: many roles in development, cancer and beyond. Cell Mol Life Sci. 2015; 72:2041-2059.

15. Wang L, Pal S, Sif S. Protein arginine methyltransferase 5 suppresses the transcription of the RB family of tumor suppressors in leukemia and lymphoma cells. Mol Cell Biol. 2008; 28:6262-6277.

16. Pal S, Baiocchi RA, Byrd JC, Grever MR, Jacob ST, Sif S. Low levels of miR-92b/96 induce PRMT5 translation and H3R8/H4R3 methylation in mantle cell lymphoma. Embo j. 2007; 26:3558-3569.

17. Hou Z, Peng H, Ayyanathan K, Yan KP, Langer EM, Longmore GD, Rauscher FJ, 3rd. The LIM protein AJUBA recruits protein arginine methyltransferase 5 to mediate SNAIL-dependent transcriptional repression. Mol Cell Biol. 2008; 28:3198-3207.

18. Pal S, Vishwanath SN, Erdjument-Bromage H, Tempst P, Sif S. Human SWI/SNF-Associated PRMT5 Methylates Histone H3 Arginine 8 and Negatively Regulates Expression of ST7 and NM23 Tumor Suppressor Genes. Molecular and Cellular Biology. 2004; 24:9630-9645.

19. Di Lorenzo A, Bedford MT. Histone arginine methylation. FEBS Letters. 2011; 585:2024-2031.

20. Kim M-S, Pinto SM, Getnet D, Nirujogi RS, Manda SS, Chaerkady R, Madugundu AK, Kelkar DS, Isserlin R, Jain S, Thomas JK, Muthusamy B, Leal-Rojas P, et al. A draft map of the human proteome. Nature. 2014; 509:575-581.

21. Branscombe TL, Frankel A, Lee JH, Cook JR, Yang Z, Pestka S, Clarke S. PRMT5 (Janus kinase-binding protein 1) catalyzes the formation of symmetric dimethylarginine residues in proteins. J Biol Chem. 2001; 276:32971-32976.

22. Guo Z, Zheng L, Xu H, Dai H, Zhou M, Pascua MR, Chen QM, Shen B. Methylation of FEN1 suppresses nearby phosphorylation and facilitates PCNA binding. Nat Chem Biol. 2010; 6:766-773.

23. Bandyopadhyay S, Harris DP, Adams GN, Lause GE, McHugh A, Tillmaand EG, Money A, Willard B, Fox PL, Dicorleto PE. HOXA9 methylation by PRMT5 is essential 
for endothelial cell expression of leukocyte adhesion molecules. Mol Cell Biol. 2012; 32:1202-1213.

24. Cho EC, Zheng S, Munro S, Liu G, Carr SM, Moehlenbrink J, Lu YC, Stimson L, Khan O, Konietzny R, McGouran J, Coutts AS, Kessler B, et al. Arginine methylation controls growth regulation by E2F-1. Embo j. 2012; 31:1785-1797.

25. He W, Ma X, Yang X, Zhao Y, Qiu J, Hang H. A role for the arginine methylation of Rad9 in checkpoint control and cellular sensitivity to DNA damage. Nucleic Acids Res. 2011; 39:4719-4727.

26. Jansson M, Durant ST, Cho E-C, Sheahan S, Edelmann M, Kessler B, La Thangue NB. Arginine methylation regulates the p53 response. Nat Cell Biol. 2008; 10:1431-1439.

27. Wei TY, Juan CC, Hisa JY, Su LJ, Lee YC, Chou HY, Chen JM, Wu YC, Chiu SC, Hsu CP, Liu KL, Yu CT. Protein arginine methyltransferase 5 is a potential oncoprotein that upregulates G1 cyclins/cyclin-dependent kinases and the phosphoinositide 3-kinase/AKT signaling cascade. Cancer science. 2012; 103:1640-1650.

28. Bao X, Zhao S, Liu T, Liu Y, Liu Y, Yang X. Overexpression of PRMT5 promotes tumor cell growth and is associated with poor disease prognosis in epithelial ovarian cancer. The journal of histochemistry and cytochemistry. 2013; 61:206-217.

29. Gu Z, Gao S, Zhang F, Wang Z, Ma W, Davis RE, Wang Z. Protein arginine methyltransferase 5 is essential for growth of lung cancer cells. Biochem J. 2012; 446:235-241.

30. Kim JM, Sohn HY, Yoon SY, Oh JH, Yang JO, Kim JH, Song KS, Rho SM, Yoo HS, Kim YS, Kim JG, Kim NS. Identification of gastric cancer-related genes using a cDNA microarray containing novel expressed sequence tags expressed in gastric cancer cells. Clin Cancer Res. 2005; 11:473-482.

31. Sinha P, Logan HL, Mendenhall WM. Human Papillomavirus, Smoking, and Head and Neck Cancer. American journal of otolaryngology. 2012; 33:130-136.

32. Thomas GR, Nadiminti H, Regalado J. Molecular predictors of clinical outcome in patients with head and neck squamous cell carcinoma. Int J Exp Pathol. 2005; 86:347-363.

33. Duffy SA, Taylor JM, Terrell JE, Islam M, Li Y, Fowler KE, Wolf GT, Teknos TN. Interleukin-6 predicts recurrence and survival among head and neck cancer patients. Cancer. 2008; 113:750-757.

34. Pak MG, Lee HW, Roh MS. High nuclear expression of protein arginine methyltransferase-5 is a potentially useful marker to estimate submucosal invasion in endoscopically resected early colorectal carcinoma. Pathol Int. 2015; 65:541-548.

35. Yang F, Wang J, Ren HY, Jin J, Wang AL, Sun LL, Diao KX, Wang EH, Mi XY. Proliferative role of TRAF4 in breast cancer by upregulating PRMT5 nuclear expression. Tumour Biol. 2015; 36:5901-5911.

36. Ibrahim R, Matsubara D, Osman W, Morikawa T, Goto A, Morita S, Ishikawa S, Aburatani H, Takai D, Nakajima J, Fukayama M, Niki T, Murakami Y. Expression of PRMT5 in lung adenocarcinoma and its significance in epithelialmesenchymal transition. Hum Pathol. 2014; 45:1397-1405.

37. Shilo K, Wu X, Sharma S, Welliver M, Duan W, VillalonaCalero M, Fukuoka J, Sif S, Baiocchi R, Hitchcock CL, Zhao W, Otterson GA. Cellular localization of protein arginine methyltransferase- 5 correlates with grade of lung tumors. Diagnostic pathology. 2013; 8:201.

38. Ghosh SK, Paik WK, Kim S. Purification and molecular identification of two protein methylases I from calf brain. Myelin basic protein- and histone-specific enzyme. J Biol Chem. 1988; 263:19024-19033.

39. Friesen WJ, Paushkin S, Wyce A, Massenet S, Pesiridis GS, Van Duyne G, Rappsilber J, Mann M, Dreyfuss G. The methylosome, a 20S complex containing JBP1 and pICln, produces dimethylarginine-modified Sm proteins. Mol Cell Biol. 2001; 21:8289-8300.

40. Jansson M, Durant ST, Cho EC, Sheahan S, Edelmann M, Kessler B, La Thangue NB. Arginine methylation regulates the p53 response. Nat Cell Biol. 2008; 10:1431-1439.

41. Yadav A, Kumar B, Datta J, Teknos TN, Kumar P. IL-6 promotes head and neck tumor metastasis by inducing epithelial-mesenchymal transition via the JAK-STAT3SNAIL signaling pathway. Mol Cancer Res. 2011.

42. Stanam A, Love-Homan L, Joseph TS, Espinosa-Cotton M, Simons AL. Upregulated interleukin-6 expression contributes to erlotinib resistance in head and neck squamous cell carcinoma. Molecular oncology. 2015; 9:1371-1383.

43. Su Y-W, Xie T-X, Sano D, Myers JN. IL-6 Stabilizes Twist and Enhances Tumor Cell Motility in Head and Neck Cancer Cells through Activation of Casein Kinase 2. PLoS ONE. 2011; 6:e19412.

44. Liu F, Zhao X, Perna F, Wang L, Koppikar P, AbdelWahab O, Harr MW, Levine RL, Xu H, Tefferi A, Deblasio A, Hatlen M, Menendez S, et al. JAK2V617FMediated Phosphorylation of PRMT5 Downregulates Its Methyltransferase Activity and Promotes Myeloproliferation. Cancer Cell. 2011; 19:283-294.

45. Ernster JA, Sciotto CG, O'Brien MM, Finch JL, Robinson LJ, Willson T, Mathews M. Rising incidence of oropharyngeal cancer and the role of oncogenic human papilloma virus. Laryngoscope. 2007; 117:2115-2128.

46. Kreimer AR, Clifford GM, Boyle P, Franceschi S. Human Papillomavirus Types in Head and Neck Squamous Cell Carcinomas Worldwide: A Systematic Review. Cancer Epidemiology Biomarkers \& Prevention. 2005; 14:467-475.

47. Chaturvedi AK, Engels EA, Pfeiffer RM, Hernandez BY, Xiao W, Kim E, Jiang B, Goodman MT, Sibug-Saber M, Cozen W, Liu L, Lynch CF, Wentzensen N, et al. Human Papillomavirus and Rising Oropharyngeal Cancer Incidence in the United States. Journal of Clinical Oncology. 2011; 29:4294-4301.

48. Kumar B, Brown NV, Swanson BJ, Schmitt AC, Old M, Ozer E, Agrawal A, Schuller DE, Teknos TN, Kumar P. High expression of myoferlin is associated with poor 
outcome in oropharyngeal squamous cell carcinoma patients and is inversely associated with HPV-status. Oncotarget. 2016; 7:18665-18677. doi: 10.18632/oncotarget.7625.

49. Aggarwal P, Vaites LP, Kim JK, Mellert H, Gurung B, Nakagawa H, Herlyn M, Hua X, Rustgi AK, McMahon SB, Diehl JA. Nuclear cyclin D1/CDK4 kinase regulates CUL4 expression and triggers neoplastic growth via activation of the PRMT5 methyltransferase. Cancer Cell. 2010; 18:329-340.
50. Brenner JC, Graham MP, Kumar B, Saunders LM, Kupfer R, Lyons RH, Bradford CR, Carey TE. Genotyping of 73 UM-SCC head and neck squamous cell carcinoma cell lines. Head \& Neck. 2010; 32:417-426. 